\title{
The Search for Design in Electrical Engineering Education
}

\author{
David V. KERNS, Sherra E. KERNS, Gill A. PRATT, Mark H. SOMERVILLE, Jill D. CRISMAN \\ F.W. Olin College of Engineering, 1735 Great Plain Avenue, Needham, MA 02492-1245 \\ david.kerns@olin.edu, sherra.kerns@olin.edu, gill.pratt@olin.edu, \\ mark.somerville@olin.edu, jill.crisman@olin.edu
}

\begin{abstract}
The importance of "design" in engineering education is well established and a cornerstone of most new engineering curricula as well as accreditation criteria. Electrical and computer engineering (ECE) programs view many elements of design in ways similar to other engineering disciplines. However, in some respects other disciplines within engineering, such as Mechanical Engineering (ME), view design in broader terms, and perhaps gain value that electrical and computer engineering educators may miss. This paper describes how design is typically viewed in ECE programs, how it's viewed in other engineering areas, particularly ME, and suggests some new possibilities for enhancing design education within ECE programs. To illustrate these possibilities, an experimental subject in which entering freshmen build a sophisticated electronic device is described.
\end{abstract}

\section{Introduction}

Most electrical and computer engineering programs view "design" as engineering exercises which are "open ended" or for which there are many possible solutions. Design problems are generally those initiated by a specification or a particular problem that needs to be solved. There is typically a wide range of possible solutions, and the form of the final solution is guided by the stated design objectives (or constraints). These objectives may be specified as requirements or limitations in cost, size, speed, etc.

Often the goal of design problems is to teach students what "real world" engineering is like. As educators, we have the notion that we will simulate in the classroom something like the experience an engineer may have in practice. There is a problem to be solved with no clear single path to the solution. The student must synthesize information from the whole of his or her past experience, integrate that knowledge in a way that suggests possible solutions, and then optimize within the set of constraints to obtain the best design. Often these projects are performed in student teams as projects to develop skills in communications, organizing and partitioning work, and other skills useful in industry.

The above characteristics of design projects are generally shared by all engineering disciplines. Now, let us focus on differences. For illustration, consider a design project recently assigned in a course on analog integrated circuit design. Teams of students were challenged to design an op-amp circuit using CMOS technology and verify its performance using SPICE. The design requirements were provided, including the required open loop gain, phase margin, gain-bandwidth product, and the challenge to use as few active devices as possible. Implicit in this assignment is an assumption that the students have had a long list of pre-requisite courses, including for example basic and perhaps advanced device physics and circuits courses. Therefore, most design projects in ECE curricula are assigned in the junior or more often senior (final) year of their undergraduate program. Often, they are part of the "capstone" design experience prescribed by ABET (Accreditation Board for Engineering and Technology). There is an assumption that all the basic fundamentals must be learned before a design is attempted. Students are pushed through a step by step process of "learning the basics", before they are allowed to exercise the creative (and much more fun) part of engineering... that of exploring, inventing, creating new solutions, new ideas, new concepts... and discovering how their creations perform.

This traditional process is a learning mode described in the educational literature as "learn-do". An excellent description of this process is presented in the book compiled by the National Research Council on "How People Learn"[1]. From an educational psychology perspective, "learn-do" requires the student to master "line by line" the material before using it. There is the alternate approach, "do-learn". It happens that a significant percentage of the population learn better in the "do-learn" mode. This approach suggests that when a student attempts a design and observes its success or failure it provides greatly enhanced motivation for the learning that follows. 
Mechanical engineering has a major branch of the discipline called design. It typically involves the creation of new and innovative mechanical systems from a set of components, without requiring a detailed understanding of the internal operation of each component. For example, a mechanical engineering design project may be to design a remote controlled vehicle. The electronic control system may be procured as a component, along with the wheels and axles. Admittedly, mechanical engineers have an advantage here, because mechanical systems are much more visible and intuitive than currents and voltages.

What is important about this approach is that these projects can be assigned to first or second year students, and they design and build something before having courses enabling complete understanding. Perhaps they design a vehicle with a chain drive, with two different size gears. They may not have yet received instruction about torque-speed curves of the motor, computed gear ratios or the fracture stress of the gear teeth, etc ... But they do design and build something. And if their gear breaks, later when they take the course on fracture stress there's a solid anchor for that knowledge. They see its value and are motivated to learn it.

The use of design projects as an implementation of the "do-learn" mode may be extremely valuable. Can "dolearn" methods be applied to learning in electrical or computer engineering education? We have recently experimented with this approach and recommend exploring it further, not as replacement for, but as a complement to existing modes of instruction.

\section{An Experimental Subject}

We conducted our experiment as a five week subject that met formally twice a week with each class lasting two hours. There were several additional hours per week of informal assistance. The student group was composed of freshmen, typically from the top of their high school classes, but with no prior experience in college physics, math or engineering. For our project we chose an optical pulse oximeter and had the students build a simplified version themselves. An optical pulse oximeter noninvasively determines a patient's blood oxygen saturation and pulse rate by measuring changes in the optical absorption spectrum of blood passing through a finger or other thin body part (e.g. an ear).

To pique the student's curiosity we first borrowed a commercial Nellcor N-200 pulse oximeter from a local hospital and allowed the students to test its operation on themselves. This fun exercise was quickly followed by posing the question: "How does this seemingly magical device work?" The students came up with various theories, all related to the obvious red light emitted by the finger clamp probe. After some speculation, a set of patents on pulse oximetry was passed out spanning some 40 years of development [2-5], as well as a fascinating paper on the recent use of similar methods to detect brain activity through the skull [6]. Starting with the oldest patent, students took turns reading out loud the summary of each one. Each reading was followed by questions from the faculty which, when answered, elucidated very satisfying "aha!"s from the rest of the class.

Half of the students were then assigned the task of studying the optical properties of oxygenated and deoxygenated hemoglobin, while the other half were given the task of studying the operation of the circuit components utilized by the simpler patents. A week later, both teams reported back to the class, and everyone had an understanding, albeit somewhat vague, of how and why the oximeter worked. This knowledge included the different absorption spectra of hemoglobin, the ability of red and infra-red light to discriminate this difference, the generation of these wavelengths by light emitting diodes, their detection (albeit with different sensitivity) by a single photo-diode, the use of synchronous detection to cancel out ambient light, and the mathematical transforms and other signal processing necessary to compensate for variations in skin thickness and color.

The remaining weeks of the subject were spent by each student experimenting with and building the subcircuits of a simplified oximeter, with students pairing up at the end to combine the sub-circuits into a final working device. Because most of today's beginning ECE students have no prior electronics experience (unlike most mechanical engineering freshmen, who have been building various structures since they were children), we utilized guided design instead of free design. In other words, in this first exercise students were given circuit topologies whose mode of operation they had to deduce, experiment with, and ultimately chose component values for to achieve the desired results.

The first circuit, shown below in figure 1 , was a hysteretic oscillator, constructed using an op-amp operating as a comparator with some positive feedback to establish the hysteresis and an RC network to establish the period of oscillation.

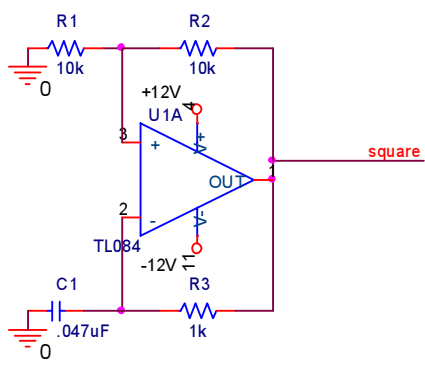


Figure 1: Hysteretic Oscillator

Given a very rudimentary description of the op-amp as a high gain device with saturation, students experimented with various values of $R 3$ and $C l$ and came to understand that it is the product of $R 3$ and $C l$ which establishes the time constant. By using analogies, the students were able to learn how the circuit worked without first learning formally Kirchoff's laws, etc ... For example; the hysteretic snap-action of the classroom light switch illuminated the hysteresis action of the circuit. A hydraulic analog of a bathtub being alternately filled and drained through a narrow pipe to two infinite reservoirs at different heights served to illustrate both qualitatively and quantitatively the relaxation of the RC network.

Next the students were introduced to negative feedback by connecting their oscillator's square wave output through a resistor to the summing junction of a second op-amp with reverse parallel LEDs (one red, one infra-red) in the feedback path, as shown in figure 2 below:

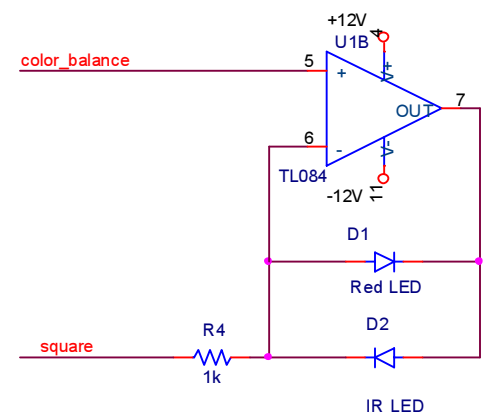

Figure 2 : LED Current Driver

Initially, the color balance wire was grounded. The red LED was on for one half of the cycle, the infra-red LED for the other half. Analogies to hydraulic feedback mechanisms in ship and car steering were used to convey an intuitive understanding of how the high open-loop gain of the op-amp was harnessed to create a low-gain transduction of voltage to current largely independent of the voltage drop of the LEDs. Students experimented with varying the gain by changing the input resistor and noting the brightness changes in the LEDs. They also noticed that the same brightness of light was emitted whether one or two LEDs were placed in series in the feedback path. A camcorder whose CCD is sensitive to IR was used to observe the operation of the infra-red LED.

With an understanding of the voltage-to-current opamp circuit, the students next built a high gain currentto-voltage circuit to detect light from a PIN photo-diode, shown in figure 3 below:

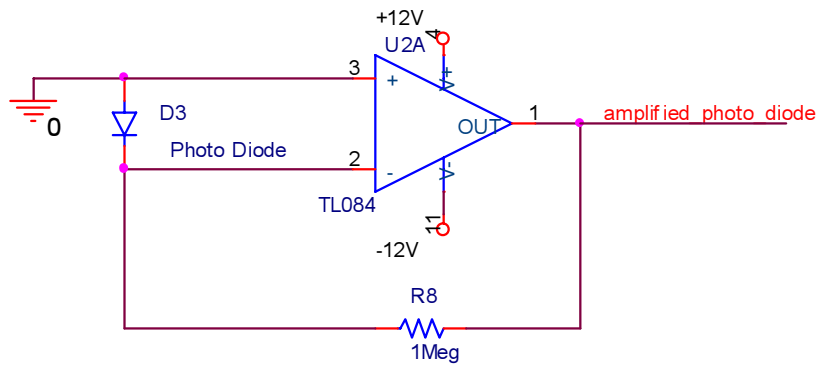

Figure 3: Photo-Diode Amplifier

Experiments with various resistor values in the feedback loop and the resulting changes in photosensitivity strengthened understanding of how gain is controlled in negative feedback circuits.

With a prior understanding from the patents of the need for synchronous detection to eliminate the effects of ambient light, the students next built a circuit with an analog switch that multiplied the output of their photodetector circuit by either +1 or -1 . As shown in figure 4 below, the analog switch was driven by the square wave output of the oscillator, so the multiplication was by -1 when the red LED was on and by +1 when the infra-red LED was on:

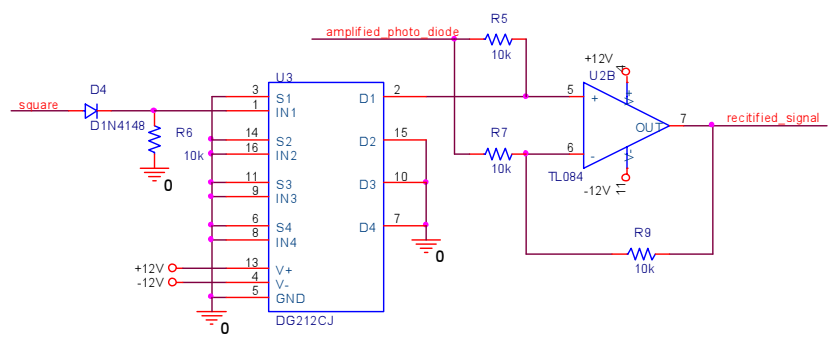

Figure $4:+1 /-1$ Multiplier

Next this signal was applied to a low-pass filter, shown in figure 5 below, to determine a signal proportional to the average difference between the light received when the red LED was on and that received when the infra-red LED was on.

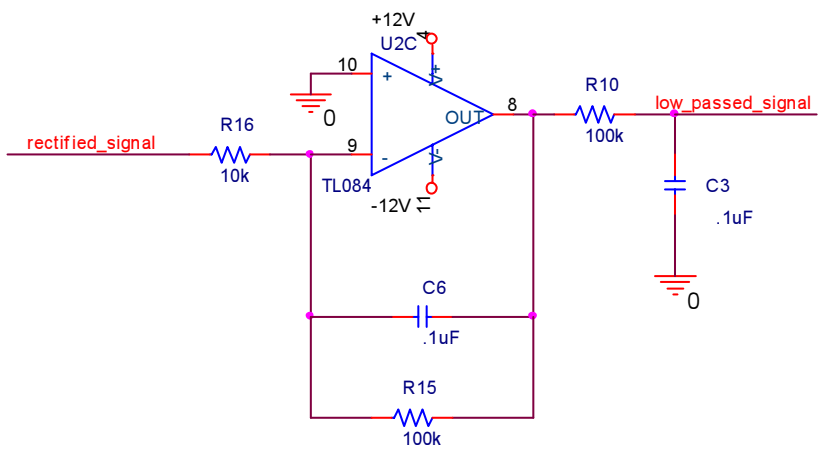

Figure 5 : First Low-Pass Filter 
As such, this signal, while not faithful to the more sophisticated formula used in the modern patents to measure oxygenation, was nevertheless monotonic in the absorption spectra changes between oxygenated and deoxygenated hemoglobin.

As shown in figure 6 below, the low-passed signal was then split into two paths.

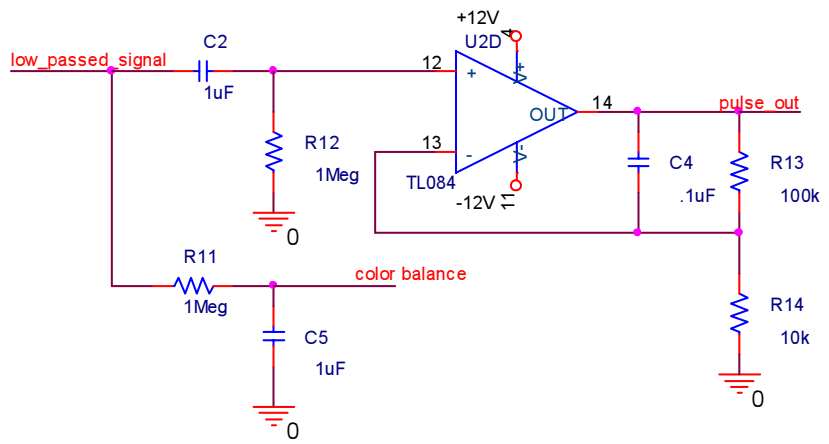

Figure 6 : Color Balance Filter \& Pulse Amplifier

It was AC coupled and further amplified and lowpassed (to further attenuate switching artifacts) and displayed on an oscilloscope as the "pulse". It was also DC coupled and further low-passed with a long time constant and fed back as a bias point for the LED voltage-to-current converter, thus controlling the brightness balance of the red vs. infra-red LEDs. This insured that the photo-diode produced, on average, the same current during each illumination half-cycle regardless of the absorption spectra of the material in between. Note that because the illumination, not the detection gain, was changed by this feedback, the synchronous detection circuit still cancelled the asynchronous signals received from ambient light. The color balance feedback signal was displayed by the students on a precision voltmeter, and served as a rough guide to the average absorption differences of the light intercepted between the LEDs and the photo-diode, i.e. the oxygen saturation. While relatively sophisticated, the students came to a good understanding of the operation of this slow outer feedback loop as they watched the intensity of the LEDs slowly change depending on the material placed in front of the photo detector.

On the final day of class, students, working in pairs, successfully combined their previous circuits to create a simplified oximeter and displayed their pulses on the oscilloscope (with amplitude varying quite widely, particularly for a student who had poor circulation, where an ear proved more successful than a finger). They noticed the time delay between their carotid pulse and that detected in their finger by the oximeter, and even competed (with a physician present, who happened to be the spouse of one of the faculty) for who could lower their saturation the farthest by holding their breath (as it turns out, the physician could).

\section{Student Reviews}

Besides being fun, this experimental subject was rated highly by the students. Averaged anonymous reviews rated the subject on a scale from 1 to 5 as shown below in table 1:

TABLE 1 : AVERAGE REVIEWS

\begin{tabular}{|l|c|}
\hline motivated me to learn & 4.4 \\
\hline helped me develop teamwork skills & 4.6 \\
\hline stimulated my intellectual curiosity & 4.6 \\
\hline was fun & 4.4 \\
\hline helped me gain new knowledge & 4.8 \\
\hline
\end{tabular}

Among the comments received were:

"I think this type of course would ... get students excited about design."

and

"I found the do-learn method a very helpful teaching style ..."

\section{What We Did Wrong}

Besides verifying our expectations of the value of "dolearn", this experiment taught us a number of important lessons about what we did wrong, and how to do a better job next time. While the "do-learn" methodology is extremely motivating and remarkably within the grasp of even beginning students, it is in fact significantly more difficult to teach this way and there is always the temptation to slide back into the easier and more familiar "learn-do" mode. We strongly believe that the increased difficulty of teaching "do-learn" is a worthwhile trade-off and leads to better student understanding, but we also learned that paying attention to the following six lessons would make the trade-off less severe.

First, "do-learn" makes early assessment difficult, and we did a poor job at this. The trouble is that most of the time students have a hazy understanding of how things work and only at the end does everything "click". This problem is similar to the issue of early assessment in "New Pathway" medical school programs where students begin working on real medical problems on day one. How do you evaluate progress when many of the early student diagnoses are likely to be wrong? We are 
currently investigating a number of ways that might address this need.

Second, we learned that "do-learn" really means a sandwich of many layers: "do-learn-do-learn ..." but since each "do" involves something that hasn't been learned yet, it is very important that the learning be very effective. "do-learn" is no substitute for good teaching.

Third, we learned that small, interactive chalk and talk (e.g. recitation) sessions, despite being conventional, are very effective, and definitely have an essential place in the "learn" part of the subject.

Fourth, we believe that having simulation available as an adjunct to experimentation would have helped a lot as it would have lessened student frustration when circuits didn't work by providing an alternate test-bed separate from implementation. We did not have the opportunity to test this in our first experiment, but will do so in the future.

Fifth, we didn't realize until the middle of the subject how important it is to always start each teaching day by explaining how the current topic fits into the larger goal of building the device. This isn't so important in "learndo" education where it is clear that a "house" is being built from the "foundation" up, but it is critical in "dolearn". We did this more towards the end of the subject, and the students were greatly relieved.

Finally, we didn't realize until mid-stream the importance of having appropriate textbook references available, because otherwise there are so many unanswered questions that frustration easily occurred. When teaching in a "do-learn" fashion, we must give students very good resources to find answers to their questions. We now realize that we need to write a new text-book (or at minimum a set of course notes) that presents the material in a manner appropriate for a "dolearn" subject. Current text books, for example, explain synchronous detectors, but use language that depends on a semester or more of ECE.

\section{Summary}

In summary, this experimental class was neither a "paint by numbers" nor "Heathkit" building exercise, nor was it a class in circuit or device theory. Rather, it was a beginning exercise in "doing" as the driver for learning. In the context of faculty guidance, each student's strong desire to build a practical device drove them to learn about the required devices and circuits in a "pull" rather than a "push" manner. It showed students that they could actually understand and build an electronic circuit that was sophisticated and useful right from the start. A piece of seemingly magical biomedical equipment had been demystified, mostly by the students themselves. We believe this is a very important lesson to inculcate in young ECE students, particularly in today's post-Ham Radio era where students typically start with very little prior experience.

Of course, this exercise is no substitute for in-depth, formal education, and we plan to take these students through formal classes in circuit and device theory. But we hope that this early "do-learn" experience, as well as others that will follow, will give our students the confidence and intuition necessary to get the most out of their advanced subjects, and help them avoid the folly of mastering the art of earning good grades without gaining a sound understanding and working knowledge of what is being taught.

We believe that obtaining the appropriate mix of "learn-do" and "do-learn" is important to facilitate learning. Creative electronic "design" projects (initially guided, as in our example, and later on less so), offered before students face rigorous technical analysis, may be one approach to enhance student motivation, understanding, and maintain an innovative spirit. At Olin College, we are experimenting with new modes of teaching. This paper has presented the results of one of our experiments in evaluating "do-learn" design projects as a vehicle for improved learning.

\section{References}

[1] National Research Council, How People Learn. Washington, D.C.: National Academy Press, 1999.

[2] J. Willam New and J. E. Corenman, "Calibrated Optical Oximeter Probe," US Patent \# 4,621,643. Nellcor Incorporated, Hayward, Calif., 1986.

[3] E. H. Wood, "Apparatus for Determining Percentage Oxygen-Saturation of Blood." US Patent \# 2,706,927. Research Corporation, N.Y., N.Y., 1955.

[4] G. A. Millikan, "Oxygen Meter." US Patent \# 2,358,992. 1944

[5] L. L. Nielsen, "Multi-wavelength incremental absorbance oximeter." US Patent \# 4,167,331. Hewlett-Packard Company, Palo Alto, CA, 1979.

[6] D. A. Benaron, S. R. Hintz, A. Villringer, D. Boas, A. Kleinschmidt, J. Frahm, C. Hirth, H. Obrig, J. C. v. Houten, E. L. Kermit, W.-F. Cheong, and D. K. Stevenson, "Noninvasive Functional Imagin of Human Brain Using Light," Journal of Cerebral Blood Flow and Metabolism, vol. 20, pp. 469-477, 2000 . 